\title{
New techniques for deriving bulge and disk models of two-component galaxies
}

\author{
R. Michard \\ 1 Observatoire de la Cote d'Azur, Dept. Augustin Fresnel, BP. 229, F-06304 Nice Cedex 4, France \\ 2 Observatoire de Paris, DEMIRM, 77 av. Denfert-rochereau, F-75014 Paris, France
}

Received December 24, 1996; accepted April 11, 1997

\begin{abstract}
A new method is introduced to "separate" the bulge and disk components of early-type galaxies, i.e. S0's and disky E's, viewed at such inclinations that their isophotes depart significantly from ellipses. In our approach, the two components are assumed to have concentric and coaxial ellipitical isophotes of arbitrary surface brightness $(\mathrm{SuBr})$ and ellipticity profiles. These four functions are to be determined by comparing calculated and easily measured quantities, i.e. the harmonics of the $\mathrm{SuBr}$ along suitable reference ellipses.

The principle of the method is straightforward and its implementation very easy in favourable cases. There are difficulties however with truly edge-on galaxies, where the assumption of elliptical isophotes is perhaps no more valid for the disk, and with objects of such inclinations that the geometrical signature of the two-components structure tends to vanish.

Our techniques have been tested with a number of calculated model objects, and with 9 real galaxies, observed at sub-arcsec resolution at the CFHT or at the Pic du Midi. The results are compared with those of other authors, sometimes involving more uncertain assumptions: the one of negligible disk contributions to the minor axis $\mathrm{SuBr}$ is unsatisfactory for S0 galaxies.
\end{abstract}

Key words: galaxies: elliptical and lenticulars, cD - galaxies: fundamental parameters galaxies: photometry

\section{Introduction}

\subsection{Brief review of previous work and outline of this contribution}

Since the pioneer work of Freeman (1970), the analysis of galaxies in terms of their bulge and disk components has

Send offprint requests to: R. Michard been a fashionable exercise. Reviews of these topics have been given by Capaccioli \& Caon (1990), and by Simien (1991).

Most of the effort has been devoted to bulge/disk decompositions based upon the fitting of ad hoc analytical formulae to the $1 \mathrm{D}$ or $2 \mathrm{D}$ surface brightness $(\mathrm{SuBr})$ distribution. Recent publications of this kind include those by de Jong \& van der Kruit (1994), Andredakis \& Sanders (1994), Byun \& Freeman (1995). Some authors however, have tried other approaches with less stringent a priori constraints. It was then necessary to consider objects projected at such inclinations to the line of sight, that the forms of the isophotes convey information about the twocomponents structure.

As an aid to derive bulge+disk models from detailed isophotal analysis, a popular approximation has been the Oblique Thin Disk, adopted by Simien \& Michard (1990), and by Scorza \& Bender (1990) and (1995). A thin disk with circular symmetry will project as a series of concentric ellipses of constant axis ratio, independently of complexities in the radial $\mathrm{SuBr}$ distribution of the disk, such as ring or lenses. This constraint will greatly help to derive the bulge and disk models. The Oblique Thin Disk approximation will possibly not remain valid if the inclination becomes so large that the intrinsic thickness of the disk, i.e. its vertical light distribution, contributes to the geometry of the isophotal contours.

Another approximation, here termed the Edge-on Very Thin Disk case, has been introduced by Capaccioli et al. (1987), and again used by Caon et al. (1990). This approach has been technically improved by Seifert \& Scorza (1996). It is then assumed that the disk is so thin, and the inclination so close to 90 degrees, that the disk does not contribute to the $\mathrm{SuBr}$ near the minor axis of the isophotes but only near their major axis. Then the bulge $\mathrm{SuBr}$ and ellipticity distributions may be recovered by fitting ellipses to limited isophotal arcs surrounding the minor axis. A bulge model is thus obtained, and the disk will be simply found by substracting this bulge model from the image. This method might give satisfactory results if the 
underlying hypothesis are valid, but unfortunately this validity cannot be tested. On the other hand, the existence of thick disks seems to be well established. According to the "quantitative morphology" of Michard \& Marchal (1993, 1994a, b) and Michard (1994), thick disks not unfrequently dominate the outer envelopes of S0's galaxies. This makes the Edge-on Very Thin Disk hypothesis quite uncertain for disk dominated lenticulars.

In the present contribution, the bulge and disk models of disky E and S0 galaxies are derived from image analysis within the assumption that both components have concentric and coaxial elliptical isophotes, with arbitrary ellipticity profiles and $\mathrm{SuBr}$ distributions. The Oblique Thin Disk approximation, or the one adopted by Kent (1986) (i.e. constant bulge and disk ellipticities) are special cases of our assumptions. Since our techniques provide complete bulge and disk models, the results can be checked by synthesising these models, adding the two corresponding images, and comparing the sum to the input galaxian image. The contribution of bulge and disk to the $\mathrm{SuBr}$ at the minor axis of an isophote can also be calculated, and the Edge-on Very Thin Disk hypothesis thus tested.

It should be emphasized however, that real galaxies do not necessarily comply with the assumptions adopted here. Even neglecting such complications as dust lanes or isophotal twists, and considering only images with two orthogonal axis of symmetry, these will not necessarily be well represented by the sum of two families of concentric and coaxials ellipses. This approximation is most likely to break in the case of edge-on, disk dominated objects.

In Sect. 2.1 are given the equations relating observable quantities to the corresponding ones, as calculated from the two-components model. These quantities are the mean value and low order even harmonics of the $\mathrm{SuBr}$ distribution along suitable reference ellipses. The important simplification occuring when these ellipses are the bulge isophotes is pointed out. In Sect. 2.2 is discussed the derivation of "external exponential models": the solution of this "restricted" problem is very useful for the derivation of an asymptotic disk model, which is a necessary ingredient of the complete solution. In the following sections are outlined our techniques for deriving and checking the complete solution: the inwards unconstrained continuation of the asymptotic model in Sect. 2.3; the improvement of the solution by introducing ad hoc constraints upon the ellipticities in Sect. 2.4; and finally the control of the solution from the $2 \mathrm{D}$ relative residuals $(\mathrm{O}-\mathrm{C}) / \mathrm{O}$.

In Sect. 3 the results of the analysis are given for 9 test galaxies covering a large range of possible inlinations and $\mathrm{D} / \mathrm{B}$ ratios. The derived models are shown graphically and commented upon in individual notes.

Finally Sect. 4 offers some comparisons with the results of other authors. A rather good agreement is found between our models and those obtained by Scorza \& Bender (1995) with the Inclined Thin Disk approximation. On the other hand the Edge-on Very Thin Disk ap- proximation, as used by Seifert \& Scorza (1996), give disk profiles in strong disagreement with our solutions, except for galaxies with an intrinsically faint disk.

\subsection{Notations and abbreviations}

It may be useful to collect here the notations and abbreviations currently used below.

. SuBr surface brightness.

. MajA major axis; MinA minor axis.

. $a, c$ major and minor axis of a Reference Ellipse, i.e. the one used in Carter's representation of isophotal contours.

. $e_{i}, f_{i}$ coefficients of cosine and sine terms in Carter's harmonic representation of deviations from the Reference Ellipse. Note that this ellipse is defined by its MajA $a$ and the conditions $e_{1}=f_{1}=e_{2}=f_{2}=0$.

. $q$ axis ratio.

. $\epsilon=1-q$ ellipticity.

. diE, boE, unE subclassification of ellipticals as disky, boxy or undeterminate; $p$ added for peculiar envelopes.

. $s p H$, thD, exD classification of envelopes, as spheroidal haloes, thick disks, extended disks respectively.

\section{Principles and implementation of the method}

The general equations relating selected observables to the looked for model are written down in Sect. 2.1 below. Various techniques for their solution might possibly be developed. The one adopted in this work involve several consecutive steps:

1. derivation of an asymptotic disk model together with associated local bulge parameters (see Sect. 2.2)

2 . inward continuation of this outer model without constraints upon the solution (see Sect. 2.3)

3. improvement of the preliminary model by introducing ad hoc constraints derived from the preceeding results (see Sect. 2.4)

4. control of the solution (see Sect. 2.5).

\subsection{General equations}

Consider an elliptical reference contour CR having the same MajA orientation as the galaxy to be analysed: its axis ratio is $q_{0}$ and its MajA length $a_{0}$. Through the tip $\mathrm{A}_{0}$ of the MajA are passing:

. one bulge isophote of $\operatorname{SuBr} I_{\mathrm{b}}\left(a_{0}\right)$ and axis ratio $q_{\mathrm{b}}$ . one disk isophote of $\operatorname{SuBr} I_{\mathrm{d}}\left(a_{0}\right)$ and axis ratio $q_{\mathrm{d}}$

Through a point $\mathrm{M}$ of the contour of eccentric anomaly $\psi$ are also passing one bulge isophote and one disk isophote. Their major axis are respectively

$a_{\mathrm{b}}(\psi)=a_{0} F\left(\nu_{\mathrm{b}}, t\right)$
$a_{\mathrm{d}}(\psi)=a_{0} F\left(\nu_{\mathrm{d}}, t\right)$ 
with the definitions $t=\tan \psi ; \nu_{\mathrm{b}}=q_{0} / q_{\mathrm{b}} ; \nu_{\mathrm{d}}=q_{0} / q_{\mathrm{d}}$ and $F(\nu, t)=\left[\left(1+\nu^{2} t^{2}\right) /\left(1+t^{2}\right)\right]^{1 / 2}$.

The contributions to the $\mathrm{SuBr}$ in $\mathrm{M}$ are respectively $I_{\mathrm{b}}\left(a_{\mathrm{b}}\right)$ and $I_{\mathrm{d}}\left(a_{\mathrm{d}}\right)$. Upon the contour RC one may measure the quantities

$$
Z_{n}=\frac{1}{2 \pi} \int_{0}^{2 \pi} I(\psi) \cos (n \psi) \mathrm{d} \psi
$$

with $n=0,2,4,6 \ldots$. These observables are related to the bulge and disk models through the equations

$Z_{n}=Z_{n, \mathrm{~b}}+Z_{n, \mathrm{~d}}$

with $n=0,2,4,6 \ldots$, where the contributions of the bulge $Z_{n, \mathrm{~b}}$ and of the disk $Z_{n, \mathrm{~d}}$ are expressed by replacing $I(\psi)$ by $I\left(a_{\mathrm{b}}(\psi)\right)$ or $I\left(a_{\mathrm{d}}(\psi)\right)$ in the integrals $(3)$.

Note that these integrals involve limited ranges of the bulge and disk models, i.e. $a_{0} q_{0} / q_{\mathrm{b}}<a_{\mathrm{b}}<a_{0}$ for the bulge, and $a_{0}<a_{\mathrm{d}}<a_{0} q_{0} / q_{\mathrm{d}}$ for the disk. These ranges are here termed the working ranges of the model.

In principle a family of reference contours could be arbitrarily selected to relate the bulge and disk models to observed quantities, provided of course that their $q_{0}$ is intermediate to the $q_{\mathrm{b}}$ and $q_{\mathrm{d}}$ at each MajA value. It is however convenient to select the (unknown!) bulge isophotal contours as reference. Then the system (4) is greatly simplified: $Z_{n, \mathrm{~b}}=I_{\mathrm{b}}\left(a_{0}\right)$ for $n=0$, and vanishes for $n \neq 0$. Also note that the equation for $n=0$ is the only one containing the bulge $\mathrm{SuBr}$; all the other equations involve only the disk model for $a>a_{0}$. Unless otherwise noted, this choice of the contour $R C$ is assumed in the following.

\subsection{The asymptotic exponential disk}

The applications of the general equations with the choice of the bulge contours for RC's, necessitates the knowledge of the disk $\mathrm{SuBr}$ and $q_{\mathrm{d}}$ profiles outside the range of $a_{0}$ where the complete representation may be sought, eventually outside the field of the available image! The quantity defined in Sect. 2.1 as "working range of a model" may indeed be much larger than $a_{0}$ if the ratio $q_{\mathrm{b}} / q_{\mathrm{d}}$ is large. This knowledge is provided by an asymptotic exponential disk model, defined by its $\mathrm{SuBr}, q_{\mathrm{d}}$ value and scale factor at the outermost attainable $a_{0}$.

If the starting $a_{0}$ is well inside an exponential disk, i.e. if the disk can be considered exponential within the working range, while the $q_{\mathrm{b}}$ variations can also be neglected, then the integrals $Z_{n, \mathrm{~d}}$ can be rewritten as

$$
Z_{n, \mathrm{~d}}=
$$

$I_{\mathrm{d}}\left(a_{0}\right) \frac{1}{2 \pi} \int_{0}^{2 \pi} \exp \left[-a_{0}\left(F\left(\nu_{\mathrm{d}}, t\right)-1\right) / a_{\mathrm{d} 0}\right] \cos (n \psi) \mathrm{d} \psi$

with $n=0,2,4 \ldots$. The quantity $a_{\mathrm{d} 0}$ is the scale factor of the disk for $a>a_{0}$. Now the equations for $n=2,4 \ldots$ contain only the four unknowns $I_{\mathrm{d}}\left(a_{0}\right), q_{\mathrm{b}}, q_{\mathrm{d}}$ and $a_{\mathrm{d} 0}$ while the equation for $n=0$ could give $I_{\mathrm{b}}\left(a_{0}\right)$ to complete the model. It has been verified that the harmonics for $n>$ 6 do not bring useful information, so that we need another equation to complete the system. This is obtained by using an auxiliairy contour of MajA $a_{0}^{\prime}$ somewhat larger than $a_{0}$. Since the disk SuBr at $a_{0}^{\prime}$ is known from $I_{\mathrm{d}}\left(a_{0}\right)$ and $a_{\mathrm{d} 0}$, this second contour adds 4 equations for $n=0,2,4 \ldots$ but no new unknown. Only one of these equations is sufficient to make the problem fully determined.

This "local exponential model" is of great practical interest, because many galaxies contain extended stretches where the disk is exponential with constant $q_{\mathrm{d}}$, while the bulge has reached a quasi constant $q_{\mathrm{b}}$. Therefore the straightforward solution of this restricted problem at one or several values of $a_{0}$, will give essential information about local values of $q_{\mathrm{b}}, q_{\mathrm{d}}, a_{\mathrm{d} 0}$ and their variations. If the outer parts of the studied galaxy contain an exponential disk, the present technique readily provides the needed asymptotic disk model.

For many galaxies however the geometrical evidence for the two-components structure disappears at some distance from the center. In this case attempts to derive an external exponential disk model do not give reliable results: tests made at several nearby values of $a_{0}$ lead to systematically varying $q_{\mathrm{b}}, q_{\mathrm{d}}$ and $a_{\mathrm{d} 0}$ solutions.

It is then necessary to select carefully the starting value of $a_{0}$ and the corresponding asymptotic model. One may be guided by Michard and Marchal's classification of the envelopes of E-S0 galaxies, derived from their Carter-like analysis. For the $s p H$ case, it is assumed that the disk vanishes in an envelope which may be formally considered as an extension of the bulge. For the exD case, the bulge becomes very faint and the envelope is an extension of the disk. for the intermediate $t h D$ case, the thick disk envelope may be considered either as an extension of the disk with a nearly vanishing bulge, or as an extension of the bulge with a vanishing thin disk. Depending upon the above cases, specific constraints have to be introduced in the determination of the asymptotic disk model to make sure it is compatible with the parameters for the one-component envelope.

Remark: The here described derivation of an external exponential disk model is one of a family of "restricted problems" involving the local representation of the model by a small set of unknowns. It would perhaps be fesible to obtain a complete general model by merging a number of such local approximate models.

\subsection{A general technique for the inward continuation of the model}

When the outer part of a disk model is known up to some $a_{1}$ value, initially as an asymptotic disk model, it will be continued inwards by selecting an $a_{0}$ contour, with $a_{0}<$ $a_{1}$, with the unknowns $q_{\mathrm{b}}\left(a_{0}\right), q_{\mathrm{d}}\left(a_{0}\right), I_{\mathrm{d}}\left(a_{0}\right)$ and $I_{\mathrm{b}}\left(a_{0}\right)$ : 
these 4 unknowns are to be derived from the 4 equations (4) with $n=0,2,4,6$. The integrals $Z_{n, \mathrm{~d}}$ can be evaluated from the known part of the disk model completed by an ad hoc interpolation for the interval between $a_{1}$ and $a_{0}$. If $a_{1}-a_{0}$ is small, the $Z_{n, \mathrm{~d}}$ integrals will be quite unsensitive to the change of the disk parameters in this range. To have a significant solution at $a_{0}$ one should take $a_{1}-a_{0}$ large enough, and interpolate back the solution to a MajA value $a_{0}^{\prime}$ closer to $a_{1}$. If however $a_{1}-a_{0}$ is large, the needed interpolation of disk parameters do not favour accuracy in the evaluation of the integrals $Z_{n, \mathrm{~d}}$.

According to these remarks, our software for continuing inwards the asymptotic model in the general case, that is without introducing constraints upon one or more of the variables, may give imprecise results, specially if one of the two components is much fainter than the other. It is however a necessary step in the present technique, because it will indicate the eventual variations of $q_{\mathrm{b}}$ and $q_{\mathrm{d}}$, and thus guide the choice of constrained, and hopefully better, solutions. It has been found useful, in order to get better estimates of the $q_{\mathrm{b}}$ and $q_{\mathrm{d}}$ profiles, to repeat the operation described here with two or more choices of the asymptotic model, starting at different $a_{0}$ values.

\subsection{Inward continuation of the model using ad hoc constraints}

To improve the solutions obtained at the preceding stage Sect. 2.3, constraints are introduced upon the run of $q_{\mathrm{d}}$, or $q_{\mathrm{b}}$, or both. These are derived from an examination and interpolation of the preliminary solutions. A number of programs have been written to continue inward an asymptotic model, with various constraints upon the axis ratio of one, or both, of the two components.

1. constant $q_{\mathrm{d}}$ as input, the remaining unknowns being $q_{\mathrm{b}}, I_{\mathrm{d}}\left(a_{0}\right)$ and $I_{\mathrm{b}}\left(a_{0}\right)$. This corresponds to the assumption described in Sect. 1.1 as the Oblique Thin Disk Model. The constant $q_{\mathrm{d}}$ is however selected as the mean of the values obtained by the general technique Sect. 2.3, not by trial and error.

2. $q_{\mathrm{d}}$ given in an input table, with the same remaining unknowns. This input table is again written from the results of stage Sect. 2.3. One of these treatments is adequate when the two components are not of too widely different $\mathrm{SuBr}$.

3. when one has to deal with a disk dominated or bulge dominated object, it is preferred to have more stringent constraints. Both $q_{\mathrm{d}}$ and $q_{\mathrm{b}}$ are then taken from an input table, again derived from the previous step, and the remaining unknowns $I_{\mathrm{d}}\left(a_{0}\right)$ and $I_{\mathrm{b}}\left(a_{0}\right)$ are taken from an ad hoc combination of the Eq. (4).

4. another algorithm has been successfully tried, where the calculated $\mathrm{SuBr}$ of the models are bound to equal the measured ones at the tips of the two major and minor axis of the reference contours.
5. a special case also considered is the assumption of constant input $q_{\mathrm{b}}$ and $q_{\mathrm{d}}$, for galaxies of moderate inclinations, with small deviations from ellipses of the observed isophotes.

\subsection{Control of the solution}

After the above operations have been performed, a complete model for both bulge and disk has been obtained, extending from the asymptotic disk model inclusive, up to an innermost MajA so selected that the region much affected by seeing or unsufficient sampling is excluded. The output file containing the $\mathrm{SuBr}$ and $q$ profiles of the bulge and disk is then used to synthesize the $2 \mathrm{D}$ image of both components, add these and compare the results to the input galaxian image. This is done by calculating a map of the $(\mathrm{O}-\mathrm{C}) / \mathrm{O}$ of local $\mathrm{SuBr}$. The solution is deemed satisfactory if these residuals are less than $10 \%$ everywhere, while they are usually much smaller near the MajA and MinA. If this is not the case, one may try to repeat the last phase of the procedure as described in Sect. 2.4, modifying the adopted $q_{\mathrm{d}}$ and/or $q_{\mathrm{b}}$ profiles and recalculating the corresponding solution.

One should also consider the possibility that the object under study cannot be well represented by the sum of a bulge and disk component, both with elliptical isophotes. This might well be the case for edge-on galaxies where the transverse and longitudinal scale lengths of the disk are not necessarily strongly coupled. One should also keep in mind the frequent occurence of boxy bulges. Finally two-components disks, as found by Seifert and Scorza for several nearly edge-on galaxies, will not necessarily be well represented by concentric ellipses. If the assumption of elliptical isophotes for the disk seems to fail, one could accept the bulge model and obtain an unconstrained disk model by substracting the assumed bulge from the galaxian image. Unfortunately there is no way to control the quality of such a solution.

Remarks: The synthesis of images required for the $2 \mathrm{D}$ test of a solution as described above is made within the contour of an ellipse enclosing the part of the image where the model is assumed valid. For aesthetical reasons, the disk and bulge parameters are extrapolated to the galaxian center in the innermost 1-3 arcsec. A routine is available to measure the elementary statistical parameters of the residuals $(\mathrm{O}-\mathrm{C}) / \mathrm{O}$ within the significant area.

\section{Models for 9 test galaxies}

\subsection{The data}

Defering the application of the present techniques to such a sample of galaxies that would be needed for deriving astrophysical results, a few objects have been selected to test the method and compare its results to previous similar studies. 
Table 1. Studied galaxies: frames and models characteristics

\begin{tabular}{lllllllll}
\hline NGC & Typ & Env & Tel & Psf & $a_{\text {ex }}$ & $a_{\text {mo }}$ & mean & $\sigma$ \\
\hline 2549 & S0 & exD & CFH & 0.59 & 35 & - & - & - \\
id & id & id & OHP & 2.06 & 132 & 82 & +2.7 & 4.6 \\
3098 & S0 & thD & CFH & 0.61 & 50 & 47 & -7.3 & 10.0 \\
3115 & S0 & spH & CFH & 0.55 & 37 & - & - & - \\
id & id & id & OHP & 3.10 & 150 & 109 & +3.1 & 4.8 \\
3245 & S0 & thD & CFH & 0.60 & 35 & - & - & - \\
id & id & id & OHP & 2.49 & 110 & 71 & +3.5 & 4.2 \\
3377 & diE & spH & CFH & 0.59 & 25 & - & - & - \\
id & id & id & CFH & - & 40 & - & - & - \\
id & id & id & OHP & 2.35 & 140 & 30 & +0.2 & 1.4 \\
3585 & diE & spH & CFH & 1.07 & 43 & 37 & -1.5 & 2.2 \\
4350 & S0 & spH & TBL & 1.38 & 86 & 66 & -3.2 & 4.2 \\
5308 & S0 & thD & TBL & 0.95 & 90 & 71 & -4.0 & 6.5 \\
5422 & S0 & thD & TBL & 0.81 & 99 & 95 & -4.0 & 7.2 \\
\hline
\end{tabular}

Table 1 lists these objects with relevant information about the used observations, and the quality of the model. All the frames were obtained in the $R$ band (Cousin's definition).

1. Column 1: NGC number of the object.

2. Column 2: Morphological type from Michard \& Marchal (1994b).

3. Column 3: Classification of the envelope from the same source.

4. Column 4: Telescope, either $\mathrm{CFH}$ for the $3.5 \mathrm{~m}$ Canada-France-Hawai telescope; TBL for the $2 \mathrm{~m}$ Bernard Lyot Pic du Midi telescope; OHP for the $120 \mathrm{~cm}$ instrument of Observatoire de Haute-Provence.

5. Column 5: FWHM of the frame PSF in arcsec.

6. Column 6: Major axis of the outermost measured isophote.

7. Column \%: Major axis of the valid range of the twocomponent model.

8. Column 8: Mean value of the model residuals $(\mathrm{O}-$ $\mathrm{C}) / \mathrm{O}$ in $\%$.

9. Column 9: Standard deviation of the same.

As may be seen from the table, the CFHT frames have a resolution of about 0.6 arcsec but a small field. The low resolution and large field frames from the OHP were used only to obtain synthetic frames by merging with the CFHT frames (see below): this is done only if the frames have a large enough range of good agreement in their respective Carter-like analysis. For edge-on disk galaxies, the OHP frames are so badly affected by seeing that the merging of data is to be avoided. The Pic du Midi frames have a resolution of circa 1 arcsec and a sufficient field. When a mixed synthetic image was used, the figures in the last three columns refer to this image.

\subsection{The use of synthetic images}

To represent a galaxy with two systems of concentric and coaxial ellipses, it is advantageous to get rid of the features in the original image that cannot comply with such a model: this includes asymmetries resulting from dust, low contrast bar or spiral structure, and isophotal twists (such as the kind described by Michard \& Marchal 1990). For this purpose, the image is analysed in terms of the representation first introduced by Carter (1978): each isophote is described by a reference mean ellipse and harmonics coefficients $e_{i}, f_{i}$ describing deviations from this ellipse. Then a modified synthetic image is built from the results of this analysis, forcing all isophotes to a common center and orientation, discarding the sine harmonics $f_{i}$ and the odd cosine harmonics $e_{2 i+1}$.

Such a synthetic image retain the essential features of the real galaxy as far as our problem is concerned, and has the advantage to be nearly devoid of noise, making more efficients the algorithms designed for its solution.

Finally if we have two images of complementary qualities for a given object, for instance a high resolution but small field image, and a lower resolution but large field one, both can be separately analysed along Carter's principles, then the resulting files intercompared and eventually mixed if they show a large enough range of good agreement. Then a synthetic image can be obtained from the mixed file, keeping the resolution of the best frame and the field of the other.

\subsection{Results}

In Fig. 1 are presented the main relevant data and the run of the bulge and disk models for the test galaxy NGC 2549. Similar figures for the 8 other test galaxies are displayed with the same captions. The data and results for each galaxy will now be commented upon.

1. NGC 2549 The data from one CFHT and one OHP frame were successfully merged to produce a synthetic "observed frame". The $\epsilon$ and $e_{4}$ coeeficients have 3 maxima, at $a$ circa 7,33 and 80 arcsec as regards $\epsilon$. Correspondingly our solution shows a disk with two bright rings and a dip in between. The bulge nearly obeys a $a^{1 / 4}$ law. In the outer range, the $q_{\mathrm{b}}$ values decreases while the $q_{\mathrm{d}}$ values increases to form a thick disk envelope. The residuals of this solution are rather small.

2. NGC 3098 It is the difficult case of an edge-on, disk dominated galaxy! The $\epsilon$ and $e_{4}$ coefficients show maxima near $a=1,10$ and $27 \operatorname{arcsec}$ (the latter two almost merging). Accordingly we find a small inner disk peaking above the main one. The range of the model is limited by the field of the CFHT frame, but the envelope has been classified $s p H$ so that the strength of the bulge component is expected to increase in the envelope. The residuals of the solution are large, possibly because the assumptions made in our technique do not well apply.

3. NGC 3115 It was studied from a synthetic frame, merging data from the CFHT and OHP frames (the merging leaves a small defect in the run of $e_{6}$ ). This 
N2549R_HMIX1
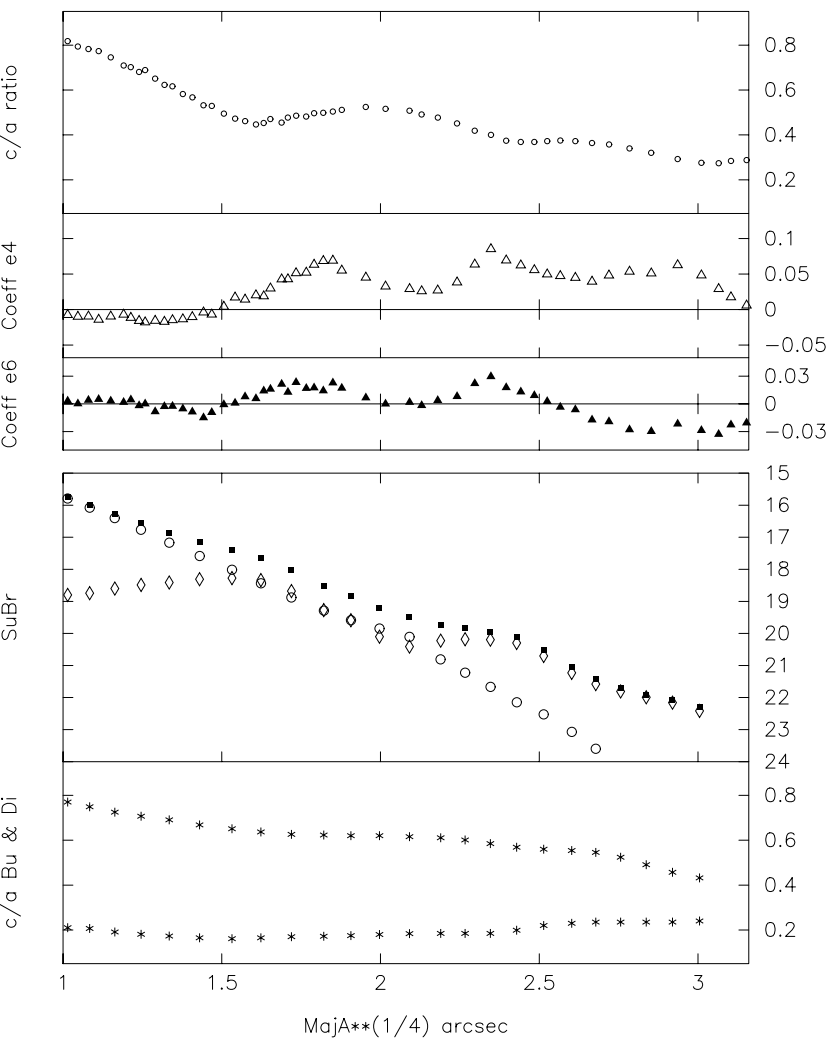

N3098R_H033

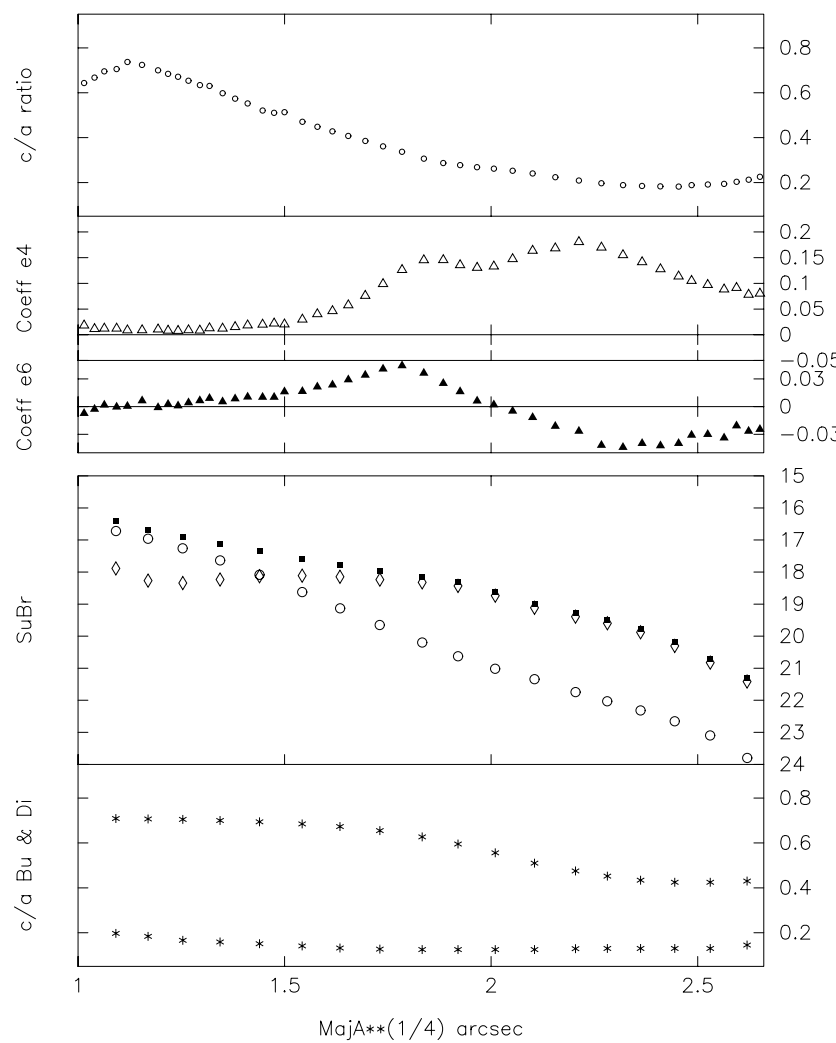

N3115R_HMIX
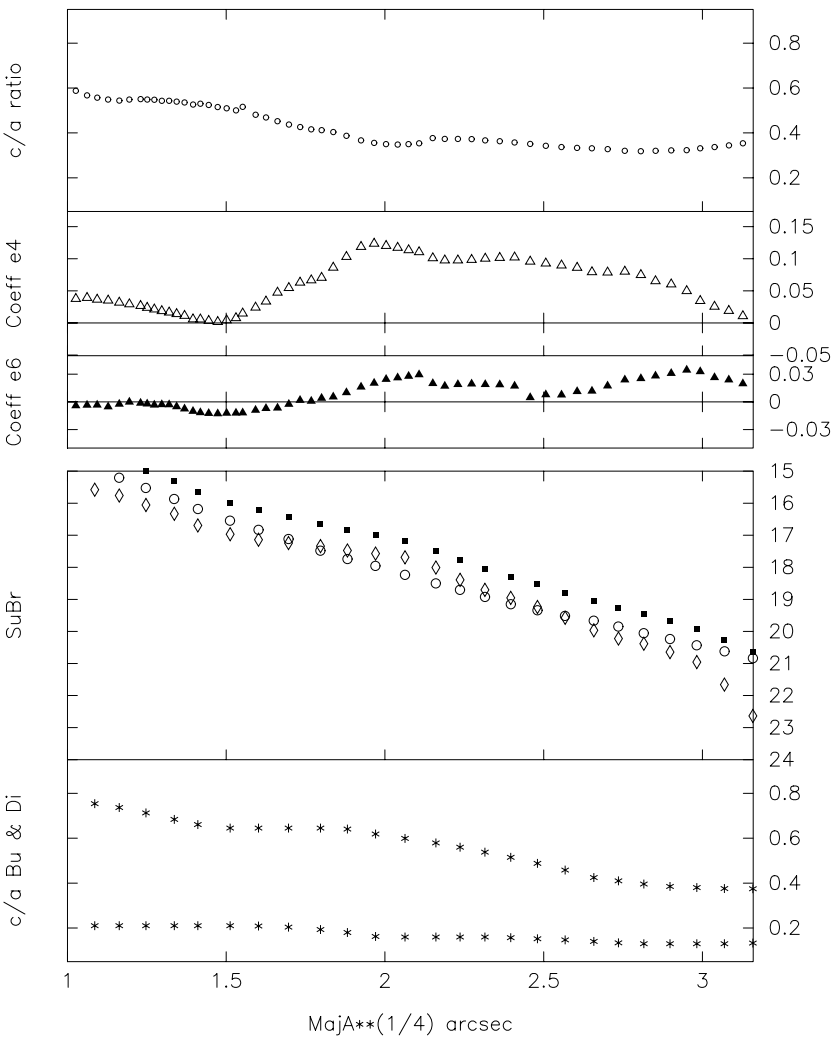

N3245R_HMIX
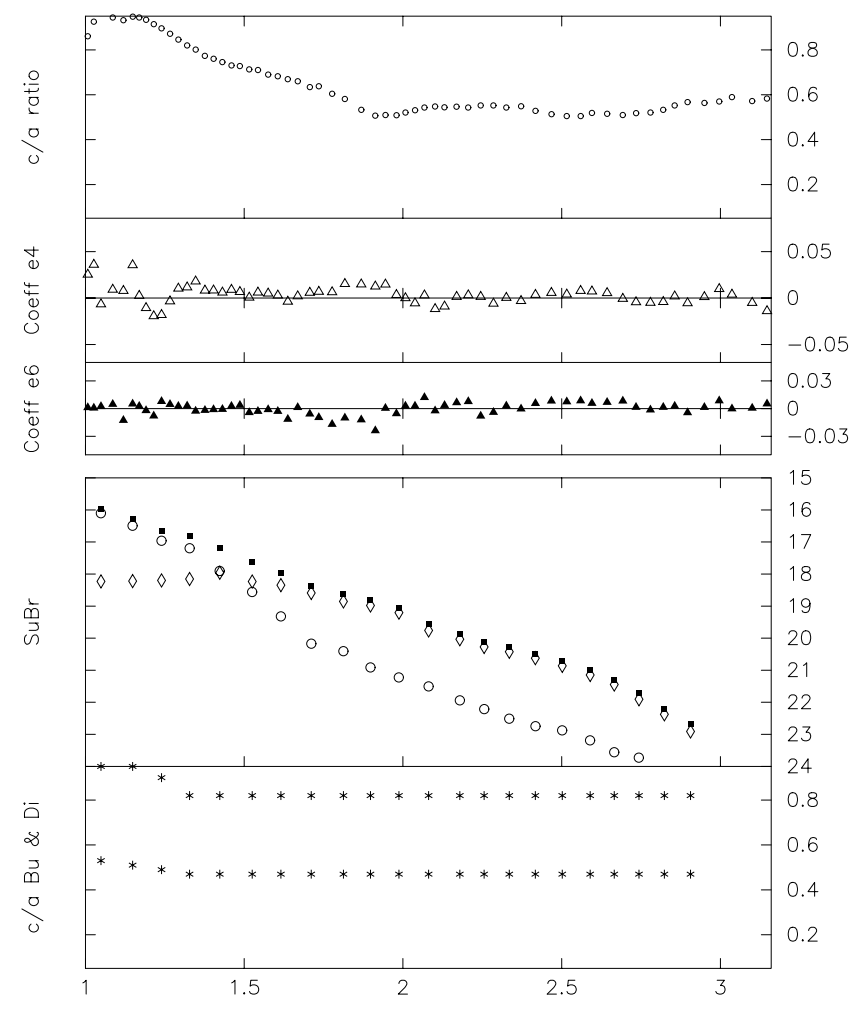

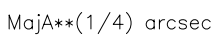

Fig. 1. The adopted abscissae are $a^{1 / 4}$ in arcsec. In the three upper windows are shown the $c / a$ ratio, the $e_{4}$ and $e_{6}$ parameters from our analysis according to Carter. The lower window gives the run of the $q_{\mathrm{b}}$ and $q_{\mathrm{d}}$ parameters. The intermediate one gives the profiles of the total $\mathrm{SuBr}$ (full squares), the bulge $\mathrm{SuBr}$ (circles) and the disk $\mathrm{SuBr}$ (spades) 
N3377R_HMIX3

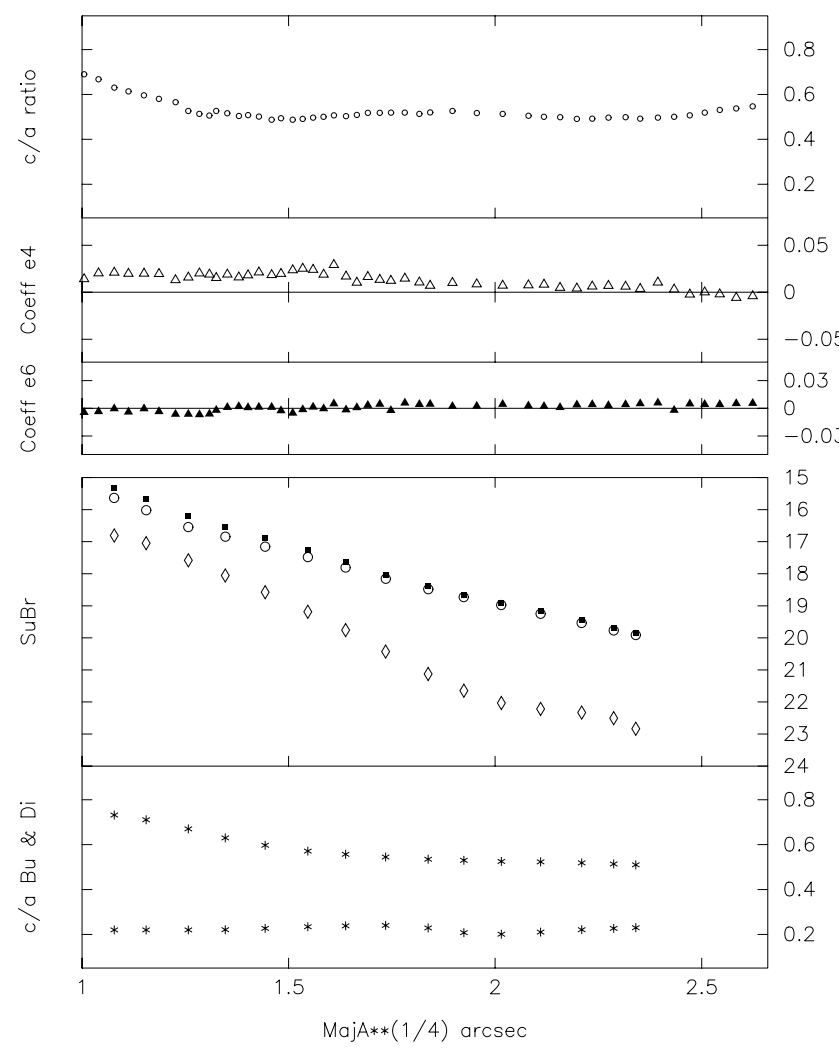

N3585R_H052

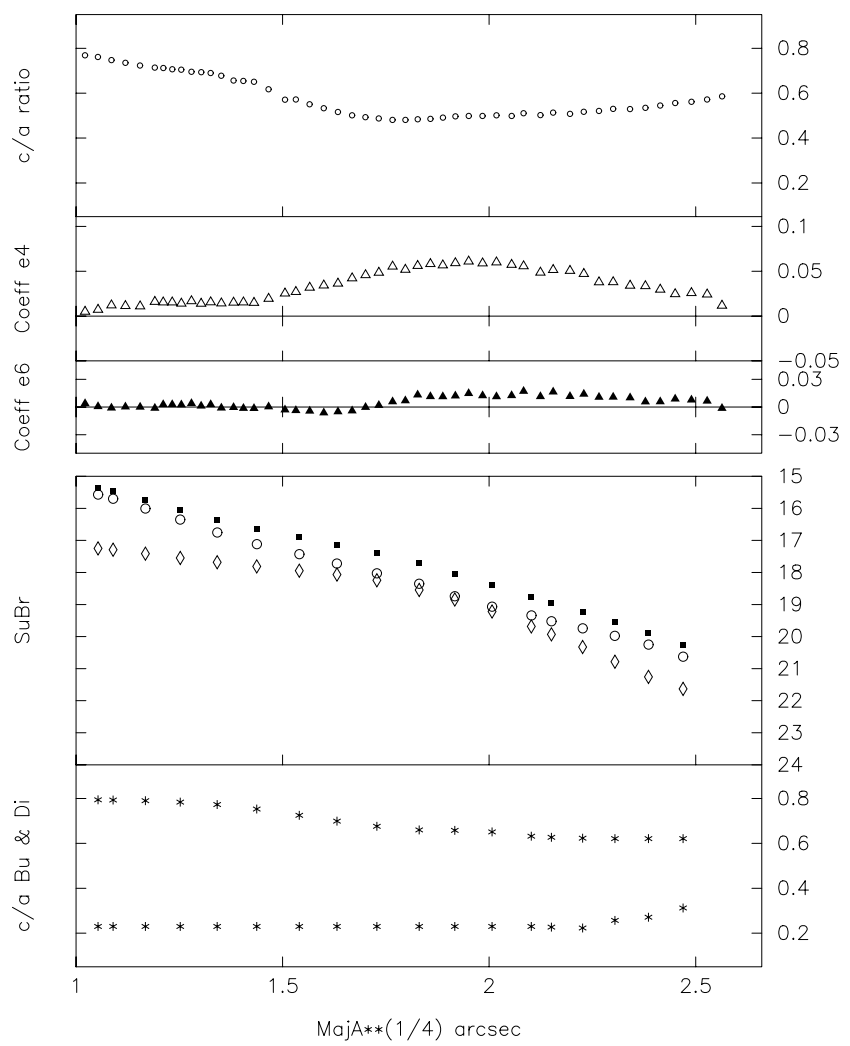

N4350R_94T314
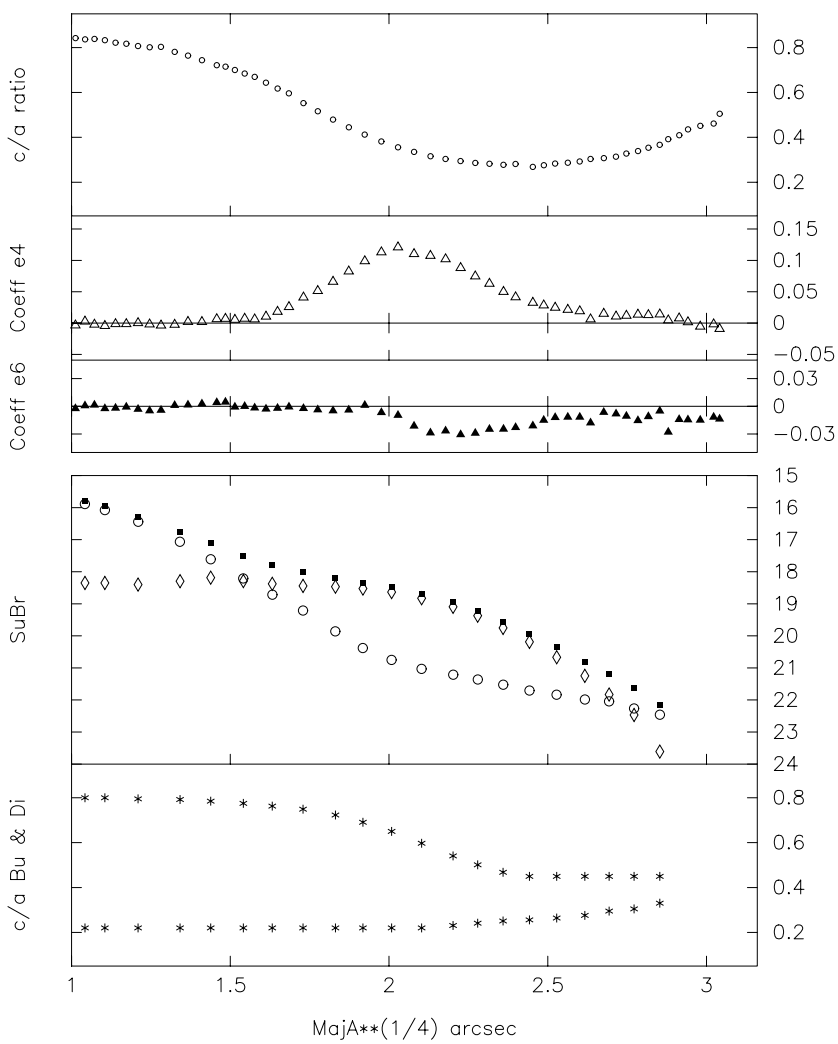

N5308R_94T122
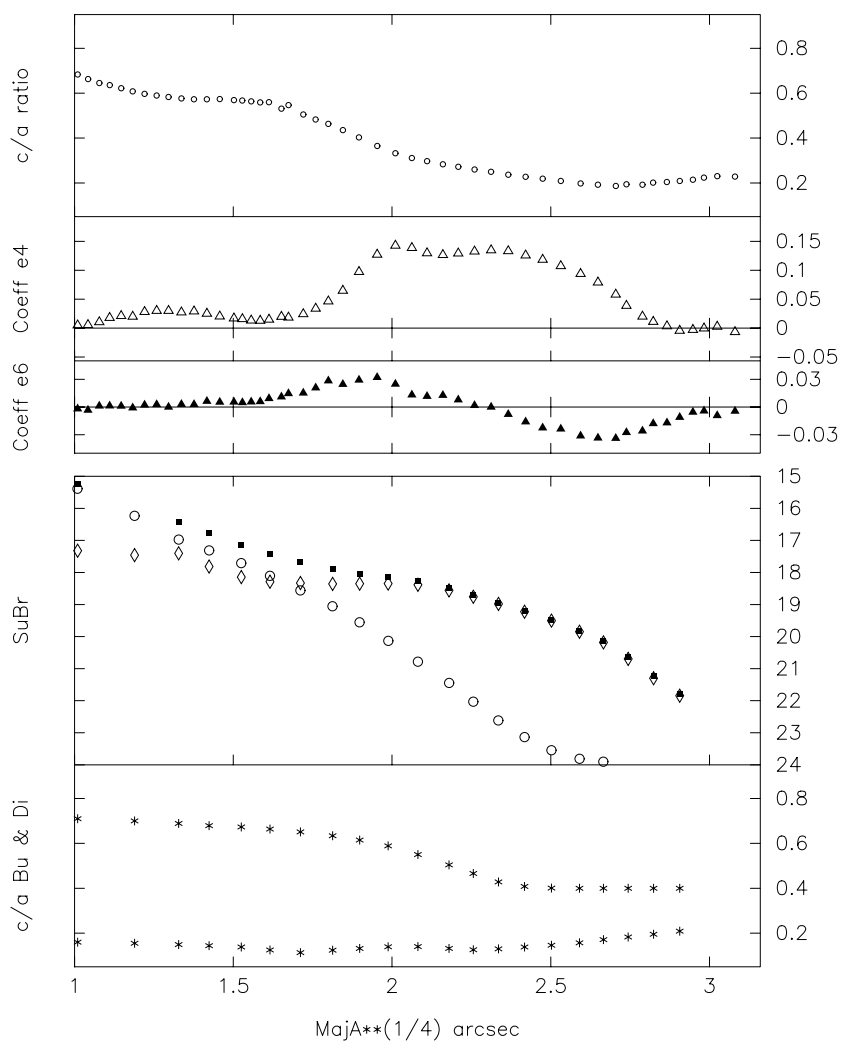

Fig. 1. continued 


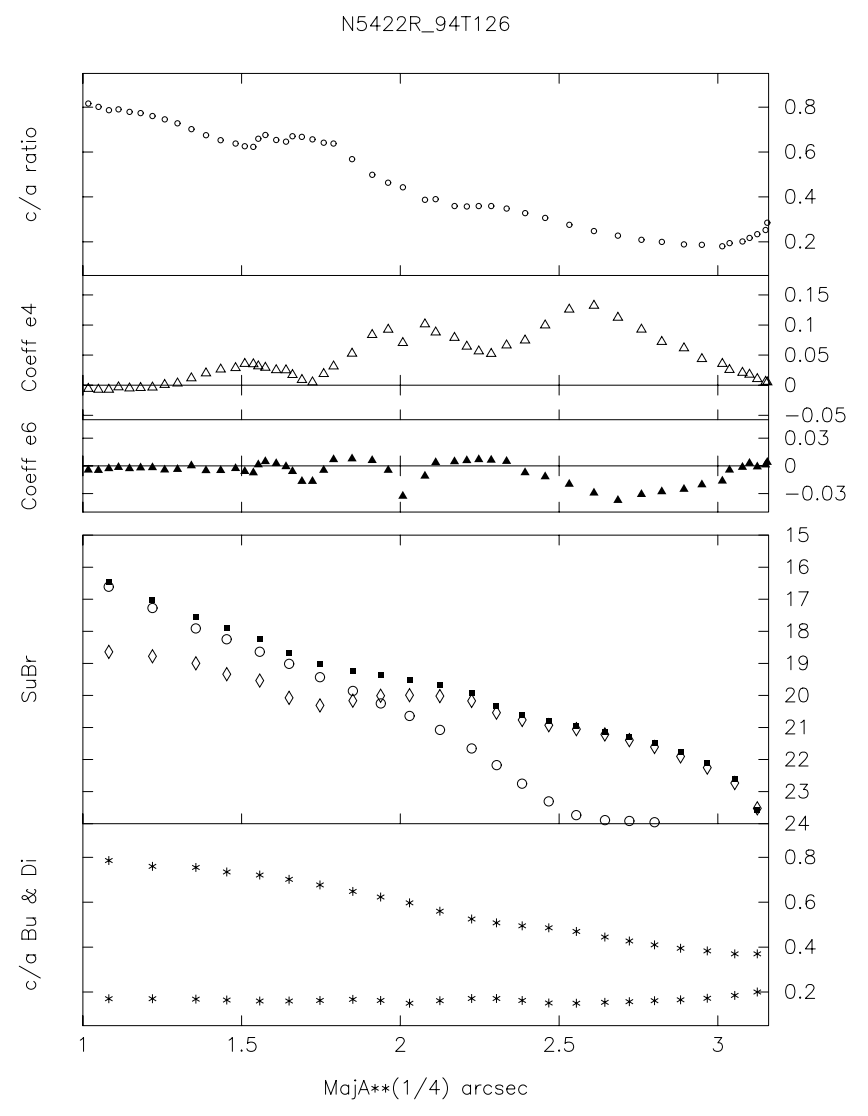

Fig. 1. continued

object has an spH envelope. The bulge follows the $a^{1 / 4}$ law. As a result of the two maxima in the $e_{4}$ coefficient, we find a bright inner disk; the main disk has a bright ring near $a=16$ arcsec, and a cut-off near $a=100$. The solution has low residuals.

4. NGC 3245 This galaxy of relatively low inclination and small Carter coefficients, was studied from a synthetic mixed frame. Approximate values of $q_{\mathrm{b}}$ and $q_{\mathrm{d}}$ were derived from local models and the complete solution was obtained with constant axis ratio for both components. The solution has low residuals, but the bulge component shows a surprising $\mathrm{SuBr}$ profile.

5. NGC 3377 This well known $d i E$ and $s p H$ object was studied from a synthetic mixed frame, involving the analysis of two CFHT and one OHP frame. In agreement with Scorza (1992) the disk has an inner bright and high gradient range, followed by a much lower gradient range and a cut-off near $a=35$ arcsec. In the high gradient range, the disk has a transverse scale height below 1 arcsec. The bulge follows the $a^{1 / 4}$ law with an inner cusp. The residuals of the solution are very small.

6. NGC 3585 This diE was studied from a CFHT frame only. The bulge follows the $a^{1 / 4}$ law. The disk has a low contrast bright ring. Our solution has very small residuals.
7. NGC 4350 is an $S 0$ with a $s p H$ envelope. The bulge is in two parts, both following the $a^{1 / 4}$ law, but with different effective scales and $q_{\mathrm{b}}$ values. It is clear that the spheroidal envelope is not simply a continuation of the inner bulge. The disk thickens prior to its cut-off near $a=70$ arcsec. The residuals for our model are small.

8. NGC 5308 is an edge-on $S 0$ with a $t h D$ envelope: as explained in Sect. 2.2 there are ambiguities in the choice of the asymptotic model. The solution was guided by assuming an outer bulge with constant $q_{\mathrm{b}}$ and a thickening disk. An inner disk well above the main disk is again found (compare with NGC 3098, NGC 3115). The residuals of our solution are relatively large.

9. NGC 5422 is a very inclined $S 0$ with a $t h D$ envelope (although there are hints of spiral structure in our unpublished $\mathrm{B}-\mathrm{R}$ maps). The $\epsilon$ and $e_{4}$ profiles show 3 maxima as in NGC 2549. In our solution, the inner bulge follows the $a^{1 / 4}$ law but its $\mathrm{SuBr}$ shows an uncertain tendency to level off in the outermost range. There is a bright inner disk, followed outwards by a bright ring, a lens...and a cut-off. As for other nearly edge-on disk galaxies, the residuals from our model are relatively large.

Table 2. Fractional disk contributions to MinA SuBr's from our models

\begin{tabular}{lllllllll}
\hline$a$ & 3 & 5 & 10 & 20 & 30 & 40 & 50 & 60 \\
\hline 2549 & 0.10 & 0.10 & 0.26 & 0.23 & 0.36 & 0.32 & 0.22 & 0.16 \\
3098 & 0.23 & 0.25 & 0.13 & 0.07 & 0.05 & - & - & - \\
3115 & 0.20 & 0.21 & 0.07 & 0.04 & 0.01 & - & - & - \\
3377 & 0.04 & 0.02 & 0.02 & 0.01 & - & - & - & - \\
4350 & 0.08 & 0.11 & 0.53 & 0.52 & 0.48 & 0.35 & 0.12 & 0.01 \\
5308 & 0.18 & 0.25 & 0.45 & 0.54 & 0.72 & 0.84 & 0.55 & 0.35 \\
5422 & 0.10 & 0.18 & 0.27 & 0.32 & 0.52 & 0.61 & 0.43 & 0.27 \\
\hline
\end{tabular}

\section{Comparison with similar work}

\subsection{Work using the Inclined Thin Disk approximation}

Because the results of Simien \& Michard (1990), were derived from photographs of relatively poor resolution, it was not found possible to make significant comparisons with the present results: indeed the observed $\epsilon, e_{4}$ and $e_{6}$ parameters in our "old" paper were obviously strongly affected by seeing for the two objects in common.

More significant comparisons are feasible for the Heidelberg results (Scorza \& Bender 1995). It is to be noted that their assumptions are similar to ours, plus the one of constant $\epsilon$, and that their criteria for finding the solution are essentially equivalent to our technique. We have three objects in common, i.e. NGC 3115, 3377 and 3585 .

As an example, the comparison for NGC 3115 is shown in Fig. 2 for the MajA total and disk SuBr's (after an ad hoc correction to bring the data to a common magnitude scale). The disk profiles are in fair agreement, although their disk is chosen flatter. Note that we both use for the 
inner region the same CFHT frame obtained by Nieto in 1989.

The agreement is still better for NGC 3585, where our $q_{\mathrm{d}}$ value is constant and nearly equal to the one of Scorza and Bender. On the other hand, the agreement is much worse for NGC 3377, probably in view of the relatively large difference in the $q_{\mathrm{d}}$ values, the galaxy beeing more inclined and the disk thinner in our model.

\subsection{Work using the Edge-on Very Thin Disk assumption}

From our models it is possible to calculate the $\mathrm{SuBr}$ at the tips of any MinA, induced by each component. The fractional $\mathrm{SuBr}$, indeed expressed in terms of the total and noted $f_{\mathrm{bu}}$ and $f_{\mathrm{di}}$, can also be obtained: their sum should be close to 1 , and one should have $f_{\text {di }}$ near zero if our models are to support the assumption that the MinA SuBr of the disk is negligible. Table 2 gives the calculated $f_{\text {di }}$ for 6 galaxies treated with this assumption by Seifert \& Scorza (1996) plus NGC 3377. The values of the fractional disk $\mathrm{SuBr}$ are given for the MinA of 9 bulge contours, specified by the values $a$ of their MajA in arcsec. It appears from Table 2, that the Edge-on Very Thin Disk assumption is valid for NGC 3377 and nearly so for NGC 3115. For the later however, the structure termed "nuclear disk" by Scorza and Bender is not very thin according to our solution, and therefore has some effect on the MinA SuBr's. The assumption is in disagreement with our models for disk dominated S0's, such as NGC 2549, 4350 (but in its outermost parts), 5308, 5422 and 3098 to a lesser extent. As a consequence, part of the MinA light, actually coming from the disk in our models, will be mistaken for bulge light in the here discussed approximation: this will lead to overestimating the bulge light and underestimating the disk. The total and disk $\mathrm{SuBr}$ profiles found by Seifert \& Scorza (1996) for NGC 2549 and 4350 are compared with our results in Fig. 3. The large and expected difference in the disk $\mathrm{SuBr}$ is striking. Note however that some specific disk features of interest (rings or "dubble disks") found in Seifert and Scorza treatment, are confirmed in our solution as seen for instance in the case of NGC 2549.

\section{Discussion and conclusion}

The separation of galaxies into their bulge and disk components from image analysis, is of course an exercise of limited physical significance. It is essentially a quantitative form of morphology, and its usefulness will have to be appreciated for the hints it may give, or constraints it may suggest, in the elaboration of physically self-consistent models.

When a galaxy is projected at a large enough inclination to the line- of-sight, the presence of the two components, their relative importance, some details of their $\mathrm{SuBr}$ profiles and the inclination of the system, are apparent from the isophotal contours and from their analysis by Carter's method, or perhaps others. This has been shown

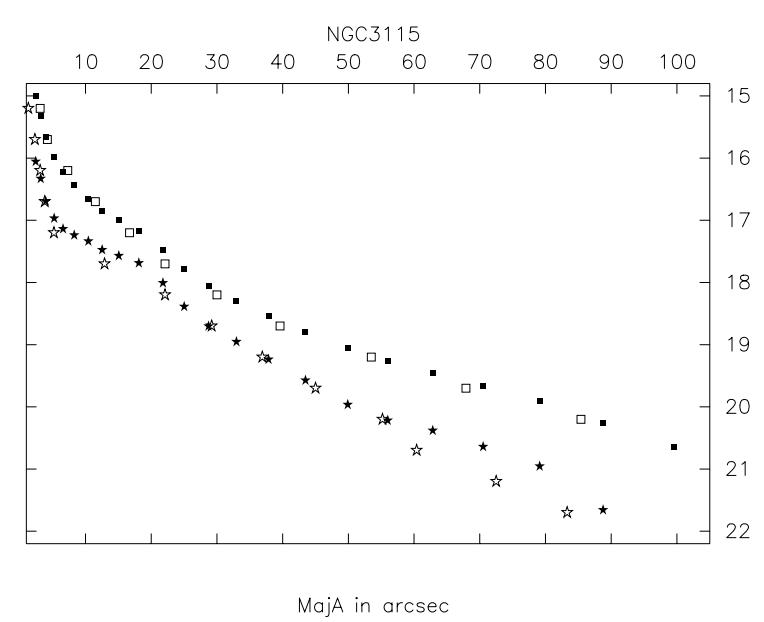

Fig. 2. Comparison between the disk profile of NGC 3115 derived by Scorza \& Bender (1995) (open symbols) and our results (full symbols). Total SuBr: squares; disk SuBr: stars

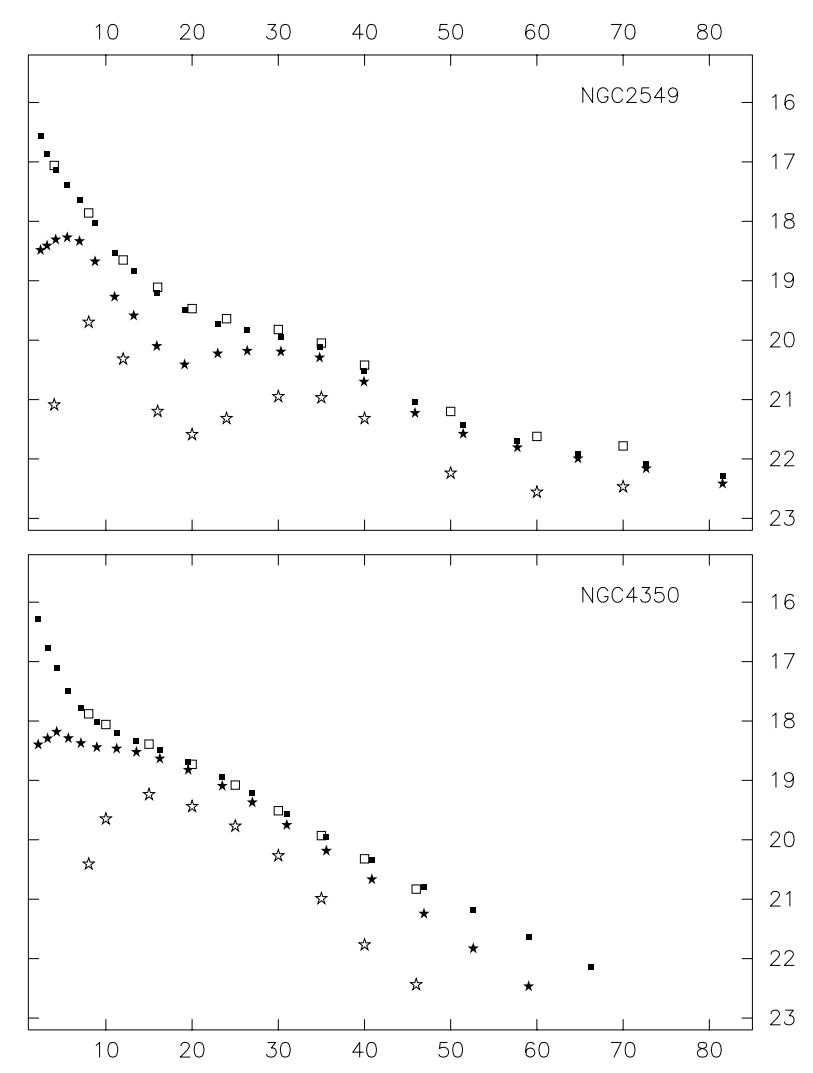

Fig. 3. Comparison between the disk profiles derived by Seifert \& Scorza (1996) (open symbols) and our results (full symbols) for two S0 galaxies. Total SuBr: squares; disk SuBr: stars 
by simulations (Rix \& White 1990), or in the numerous papers quoted above in Sect. 1.1. It is then of interest to derive two-components galaxian models from some form of isophotal analysis of the image.

In our contribution to this approach, it was proven feasible to relax part of the approximations made in earlier work: it is here assumed only that the bulge and disk project are two systems of coaxial and concentric ellipses. The $\mathrm{SuBr}$ and $\epsilon$ profiles of the two system are not a priori constrained.

The problem of deriving these four unknown functions from image analysis could perhaps be tackled more efficiently than by the step by step procedure described in Sect. 2, It should be emphasized however than any approach will involve the derivation of a necessarily uncertain asymptotic model, and various alternatives to adapt the number of degree of freedoms to the available information: this heavily depends upon the inclination (too large or too small, it leads to difficulties!) and to the relative "strengths" of the bulge and disk (if one dominates too much uncertainties occur).

As it is, the techniques here presented have the following advantages:

1. They give, as explained in Sect. 2.3, a direct insight into the profiles of the axis ratios $q_{\mathrm{b}}$ and $q_{\mathrm{d}}$, allowing a choice of plausible interpolated profiles for these quantities, including the "classical" one $q_{\mathrm{d}}=$ cte.

2. They provide a complete model for bulge and disk, allowing to calculate their respective contributions to the galaxian image at any position, and providing a stringent $2 \mathrm{D}$ test of the formal quality of the representation.

3. When the assumptions underlying our techniques do not lead to good solution, i.e. when the galaxian image cannot be well represented by two systems of elliptical isophotes, we are still left with an approximate bulge model, to be substracted from the image to provide an approximate disk model.

After experimenting with 9 galaxies of various characteristics, and considering also the results of previous work, it seems to us that the derivation of two-components models for galaxies at suitable inclinations, may lead to interesting indications about the followings topics:

1. the light profile of bulges, often very close to the $r^{1 / 4}$ law, but with possibly significant deviations in some cases (NGC 4350 and other S0's with spH envelopes?)

2. the light profiles of disks, with the structure of bright and dark rings, or lenses, that is found in all the studied S0's, although at various degrees of contrast,

3 . the intrinsic thickness of disks, which for near edgeon galaxies, can be approached from their transverse scale-heights,

4. the colour contrast between a bulge and a disk, which could be more prcisely measured after their "separation" (see an example by Silva et al. 1989).
The present techniques might be perhaps applied to some spirals galaxies, provided their images can be cleaned from the troublesome effect of localized dust and star formation regions (possibly through observations in selected IR bands?). Only spirals where the projected disk does not largely deviate from an ellipse could be considered.

One should finally comment that poor seeing can lead to completely spurious results in the type of image analysis here considered. In the inner galaxian regions, the MajA light from the disk will be transfered to its MinA, and added to the bulge light. Eventually, a spurious hole in the disk light may result! Farther out, the contrast of an edge-on thin disk might be much reduced by seeing, as we experinced by comparing frames of NGC 3377, 3115, 3098, 4350. 5308... obtained at various resolutions.

Acknowledgements. Part of the frames used in this work where obtained by J.L. Nieto at the CFHT and J. Marchal at the OHP. I geatly benefited from their cooperation and their friendship until their untimely death. Dr. P. Poulain obtained the other CFHT and Pic du Midi frames as part of a cooperative study of colour distributions in early-type galaxies: I am glad for his permission to use this material in the present work.

\section{References}

Andredakis Y.C., Sanders R.H., 1994, MNRAS 267, 283

Byun Y.I., Freeman K.C., 1995, ApJ 448, 563

Caon N., Capaccioli M., Rampazzo R., 1990, A\&AS 86, 429

Capaccioli M., Caon N., 1990, in: Morphological and Physical Classifications of Galaxies, Proc. of the 5th Int. Worshop of the Osserv. Astr. di Capodimonte, Sant'Agata sue due Golfi, Sep. 3-7 1990. Kluwer Publ. (1992)

Capaccioli M., Held E.V., Nieto J.L., 1987, AJ 94, 1519

Carter D., 1978, MNRAS 182, 797

Freeman K., 1970, ApJ 160, 811

Kent S.M., 1986, AJ 91, 1301

de Jong R.S., van der Kruit P.C., 1994, A\&AS 106, 451

Michard R., 1994, A\&A 288, 401

Michard R., Marchal J., 1990, A\&A 232, L9

Michard R., Marchal J., 1993, A\&AS 98, 29

Michard R., Marchal J., 1994, A\&A 283, 779

Michard R., Marchal J., 1994, A\&AS 105, 481

Rix H.W., White S.D.M., 1990, ApJ 362, 52

Scorza C., 1992, in: Structure, Dynamics and Chemical Evolution of Elliptical Galaxies, ESO/EIPC Workshop, Marciana Marina, Elba, 25-30 May 1992, Proceedings, Danziger I.J, Zeilinger W.W. and Kjär K. (eds.). ESO Publ. (1993) p. 115

Scorza C., Bender R., 1990, A\&A 235, 49

Scorza C., Bender R., 1995, A\&A 299, 20

Seifert W., Scorza C., 1996, A\&A 310, 75

Silva D.R., Boroson T.A., Thompson I.B., Jedrzejewski R.I, 1989, AJ 98, 131

Simien F., 1991, in: 3rd ESO/ST-ECF Data Analysis Worshop, Garching, 22-23 Avr. 1991, Proceedings, Grosbol P.J. and Wormels R.H. (eds.). ESO Publ. (1992)

Simien F., Michard M., 1990, A\&A 227, 11 